\section{Preoccupation with one's appearance: a motivating factor for cosmetic dental treatment?}

\author{
A. De Jongh, ${ }^{1}$ F. M. D. Oosterink, ${ }^{2}$ Y. R. van Rood ${ }^{3}$ and I. H. A. Aartman ${ }^{4}$
}

IN BRIEF
- Tooth whitening seems to have become
the most popular cosmetic dental
treatment in recent years.
There is no reason to assume that Body
Dysmorphic Disorder (BDD) plays a
significant role in the majority of people
who seek cosmetic dental care.
Dentists should be aware that being
dissatisfied with one's own appearance
seems to be an important reason to seek
cosmetic dental treatment.

\begin{abstract}
Background It has been estimated that among patients presenting for cosmetic treatments up to $15 \%$ suffer from Body Dysmorphic Disorder (BDD), a psychiatric condition characterised by a preoccupation with an imagined defect in appearance. Objectives The main purpose of the current study was to establish the relationship between presence of BDD characteristics and interest in aesthetically motivated dental treatments. Methods Data were obtained by means of a survey within a sample of 879 Dutch citizens of 16 years and older. Characteristics of BDD were assessed based on DSM-IV criteria. Results Only one of the BDD features (ie a preoccupation with a defect of appearance) emerged as a significant predictor of undergoing cosmetic dental treatments. Patients with such preoccupation were nine times more likely to consider tooth whitening, and six times more likely to consider orthodontic treatment. They were also five times more likely to be dissatisfied about their most recent treatment. Conclusions The results suggest that preoccupation with one's physical appearance is a motivating factor for undergoing certain types of cosmetic dental procedures.
\end{abstract}

\section{INTRODUCTION}

Undergoing cosmetic treatment to improve physical appearance has become increasingly popular. This interest can likely be attributed to several factors, including cultural images of beauty, media attention through television programs and health and beauty magazines, as well as a more accepting attitude regarding cosmetic medical treatments as a means of physical enhancement. ${ }^{1}$ According to the American Society of Aesthetic Plastic Surgery more than 11.5 million cosmetic surgical (eg liposuction, breast augmentation) and nonsurgical cosmetic procedures (eg botox injections, laser hair removals) were performed in 2006, which represents an increase of $446 \%$ since $1997 .^{2}$

\footnotetext{
1*2,4 Department of Social Dentistry and Behavioural Sciences, Academic Centre for Dentistry Amsterdam, Universiteit van Amsterdam, Louwesweg 1, 1066 EA Amsterdam, The Netherlands; ${ }^{3}$ Department of Psychiatry, Leiden University Medical Centre, the Netherlands ${ }^{*}$ Correspondence to: Ad de Jongh Email: a.de.jongh@acta.nl
}

\section{Refereed Paper}

Accepted 14 November 2007

DOI: $10.1038 /$ sj.bdj.2008.522

${ }^{\circledR}$ British Dental Journal 2008; 691-695
It has been estimated that among patients presenting for cosmetic treatments up to $15 \%$ suffer from a psychiatric condition termed Body Dysmorphic Disorder (BDD) also known as dysmorphophobia, literally meaning 'fear of ugliness'., ${ }^{3,4}$ BDD has an estimated prevalence of 1 to $2 \%$ in the general population and is characterised by a preoccupation with a slight or imagined defect with some aspect of physical appearance..$^{5-8}$ BDD is marked by severe disruptions of self-esteem, avoidance of social situations, a high level of suicide attempts and a tendency to seek non-psychiatric medical or surgical treatment to improve the imagined defect in appearance. ${ }^{8-10}$ Although BDD is a psychiatric disorder, rather than seeking help for their psychological problems, most affected patients present to dermatologists or cosmetic surgeons to improve their perceived defect. ${ }^{11}$ However, there are indications that such treatments usually prove unsatisfactory, both to the patient and the practitioner. ${ }^{11,12}$

Until now, empirical studies on the prevalence of BDD have mainly been focussed on the rate of BDD among cosmetic surgery and dermatology populations. $^{4,13}$ Only two studies have investigated the rate of BDD among patients presenting for non-surgical cosmetic procedures, both pertaining to procedures like botox injections and chemical peels. ${ }^{3}$ Although a few case descriptions of BDD have also been reported in dental practices ${ }^{14,15}$ and maxillofacial surgery clinics ${ }^{16-18}$ the prevalence rate of BDD patients who seek aesthetically motivated dental treatment is unknown.

Given the growing interest in cosmetic procedures the question arises whether patients with symptoms typical of BDD are particularly overrepresented among persons who seek cosmetic enhancement through dental treatment. Accordingly, the main purpose of the present population-based study was to establish the relationship between presence of characteristics of BDD and interest in cosmetic dental treatments. Since there is evidence to suggest that cosmetic procedures typically do not improve the appearance concerns of individuals with symptoms of BDD and, in some cases, may exacerbate symptoms, it 
was postulated that people with BDD characteristics are more likely to be dissatisfied with previous treatments. An additional aim included predicting interest in aesthetically motivated dental treatment using sociodemographic variables and BDD characteristics as independent variables.

\section{METHOD}

\section{Subjects}

Between April and June 2005, 1,522 people were randomly approached by two advanced graduate students at several locations, and were asked whether they would be willing to participate in a study on health and physical appearance. The locations were public places (eg supermarkets, terraces, parks and shopping malls, etc) previously selected to provide a geographically diverse sample (eg both urban and rural areas) and sufficient opportunity to complete the questionnaire. If the potential participant agreed to participate, was over 16years-old, and had sufficient command of the written Dutch language, s/he was requested to fill out a questionnaire involving a number of health-related subjects. Of all people approached 906 (60\%) agreed to participate.

\section{Procedure}

The research was approved by and carried out under the auspices of The Netherlands Institute for Dental Sciences (IOT). The questionnaire developed for the current study contained items pertaining to demographic variables (ie age, gender, marital status, and race/ethnicity), history of cosmetic treatments (ie jaw correction operations, orthodontic treatments, crowns, facings, whitening, and combinations of these treatments) and intentions to undergo these treatments. The question to determine the rate of subjects who had undergone a cosmetic dental treatment in the past was: 'Did you ever undergo a cosmetic dental procedure to improve the appearance of your anterior teeth?' If the answer was in the affirmative the next question was: 'Which type of cosmetic procedure did it concern?' Similar questions were asked about future cosmetic dental procedures. Respondents were further asked to indicate the extent to which they were satisfied with their most recent cosmetic treatment, on a three point Likert-type scale ('dissatisfied or very dissatisfied', 'not satisfied, but also not dissatisfied', 'satisfied to very satisfied').

To assess features of BDD the following criteria of the Diagnostic and Statistical Manual for Mental Disorders (DSM IV-TR $^{4}$ ) were used:

I. Preoccupation with an imagined defect in appearance. If a slight physical anomaly is present, the person's concern is markedly excessive

II. The preoccupation causes clinically significant distress or impairment in social, occupational, or other important areas of functioning.

Preoccupation with a defect in appearance was assumed present if the respondent answered the following question in the affirmative: 'Are you preoccupied with the idea that your body or a part of your body is unattractive, ugly, deformed or not beautiful enough?' When this question was answered in the negative, the patient could not fulfil the criteria for BDD and therefore further questions were skipped. Those who answered the first question in the affirmative got four more questions to answer. Firstly, to obtain a conservative check as to whether or not the defect in appearance is mainly or solely the perception of the respondent (an 'imagined defect') the following screening question was used: 'Do you remain convinced that your body or a part of your body is unattractive, ugly, deformed or not beautiful enough even though others are convinced that this is not true?' Secondly, three additional questions assessed distress or impairment in social (eg 'Does the preoccupation with your appearance cause distress or does it interfere in the way you relate to others?'), occupational or other important areas of functioning. The combination of being preoccupied with the idea of a defect in appearance, holding on to the conviction of being unattractive despite the fact that others say that this is not true, and reporting distress related to the preoccupation or interference in at least one area of daily functioning was considered to be a positive diagnostic screen for BDD (a field trial investigating the use of these items as a screening instrument for BDD against the Structured Clinical Interview for DSM-IV [MINI+] as gold standard for the detection of BDD revealed excellent sensitivity [1.0] and very good specificity [0.92]). ${ }^{19}$

\section{Statistical analyses}

The strength of the associations between report of cosmetic treatments and the independent variables (sociodemographic variables and BDD characteristics) was measured by the odds ratio (95\% confidence intervals are reported). Multivariable logistic regression analyses were used to examine the relationship between interest in cosmetic dental treatments (past treatments or future plans) and the independent variables (sociodemographic variables, previous cosmetic treatments, and BDD characteristics), while controlling for all other factors in the model. The Hosmer-Lemeshow goodness-of-fit chi-square statistics were calculated to evaluate how well the data fit the models.

\section{RESULTS}

\section{General descriptive data of the participants}

Of the 906 completed questionnaires 27 could not be used due to missing data and were removed from further data analyses. The age of the 879 remaining respondents (55\% women) varied between 16 and 84 years $(\mathrm{M}=33.2$; $\mathrm{SD}=$ 13.7). The distribution of gender, marital status and race/ethnicity of the sample was comparable with the figures published by the Dutch Central Bureau of Statistics in the Netherlands. ${ }^{20}$

\section{Cosmetic dental treatments in the past and in the future}

About a quarter ( $n=217 ; 24.9 \%$ ) of the respondents reported to have undergone one or more cosmetic dental treatment(s) in the past. No gender difference was found. Relatively young individuals (ie $<30$ years) were significantly more likely than those who were older to have a history of cosmetic dental treatments [30.3\% versus $19.4 \%$; OR $=1.8 ; 95 \% \mathrm{CI}$, 1.3-2.5]. As shown in Table 1 two most 
Table 1 Report of past and planned cosmetic dental procedures and strength of associations (OR) between the presence of a preoccupation with one's physical appearance and treatment type

\begin{tabular}{|c|c|c|c|c|c|c|c|c|}
\hline \multirow[b]{2}{*}{ Type of treatment } & \multicolumn{4}{|c|}{ Past treatments } & \multicolumn{4}{|c|}{ Planned treatments } \\
\hline & $\mathrm{N}$ & $\%$ & $\begin{array}{l}\% \text { of } \\
\text { total } \\
\text { sample }\end{array}$ & $\begin{array}{l}\text { OR } \\
(95 \% \\
\mathrm{Cl})\end{array}$ & $\mathrm{N}$ & $\%$ & $\begin{array}{l}\% \text { of } \\
\text { total } \\
\text { sample }\end{array}$ & $\begin{array}{l}\text { OR } \\
(95 \% \mathrm{Cl})\end{array}$ \\
\hline Whitening of teeth & 7 & 3.3 & 0.8 & n.s. & 32 & 40.3 & 3.2 & $\begin{array}{l}9.2(4.1- \\
20.8)^{* * *}\end{array}$ \\
\hline Orthodontic treatment & 124 & 53.3 & 13.1 & n.s. & 13 & 19.4 & 1.5 & $\begin{array}{l}6.0(1.8- \\
20.6)^{* * * *}\end{array}$ \\
\hline Crowns & 50 & 23.4 & 5.8 & n.s. & 11 & 16.4 & 1.3 & n.s. \\
\hline Facings & 1 & 0.5 & 0.1 & ** & 2 & 3.0 & 0.2 & **. \\
\hline $\begin{array}{l}\text { Surgical correction } \\
\text { of the jaw(s) }\end{array}$ & 0 & 0.0 & 0.0 & ${ }^{* *}$ & 1 & 1.5 & 0.1 & ** \\
\hline Combinations & 30 & 13.9 & 3.4 & n.s. & 4 & 6.0 & 0.4 & ** \\
\hline Other types of treatments* & 12 & 5.6 & 1.4 & n.s. & 9 & 13.4 & 1.1 & ** \\
\hline \multicolumn{9}{|c|}{$\begin{array}{l}\text { OR }=\text { odds ratio; } \mathrm{Cl}=\text { confidence interval; } \mathrm{n} . \mathrm{s}=\text { = non significant, } \mathrm{p}>0.05 \\
{ }^{*} \text { Mainly composite restorations and changes of shape } \\
{ }^{* *} \text { Odds ratios could not be computed due to a low prevalence rate or an empty cell } \\
* * * p=0.0001\end{array}$} \\
\hline
\end{tabular}

Table 2 Prevalence of symptoms of BDD among patients with and without the intention to undergo cosmetic dental treatment within the coming year and strength of associations $(\mathrm{OR})$ between the presence of BDD characteristics and treatment intention

\begin{tabular}{l|c|c|c|c|c|c}
\hline & \multicolumn{7}{|c}{$\begin{array}{c}\text { Intention to undergo cosmetic dental treatment } \\
\text { within the coming year }\end{array}$} \\
\cline { 2 - 7 } & \multicolumn{2}{|c|}{$\begin{array}{c}\text { Yes } \\
(n=73)\end{array}$} & \multicolumn{2}{c|}{$\begin{array}{c}\text { No } \\
(n=782)\end{array}$} \\
\cline { 2 - 7 } & $\mathrm{N}$ & $\%$ & $\mathrm{~N}$ & $\%$ & OR & $95 \% \mathrm{Cl}$ \\
\hline $\begin{array}{l}\text { Preoccupation with one's } \\
\text { physical appearance }\end{array}$ & 22 & 30.1 & 48 & 6.1 & 6.6 & $3.7-11.8$ \\
\hline $\begin{array}{l}\text { Convinced of unattractiveness } \\
\text { despite the opposite conviction } \\
\text { of others }\end{array}$ & 15 & 67.3 & 33 & 68.2 & 1.0 & $0.3-3.5$ \\
\hline Marked distress or impairment & 6 & 27.3 & 8 & 18.4 & 1.7 & $0.5-5.4$ \\
\hline Full diagnostic screen of BDD & 4 & 18.4 & 9 & 18.2 & 0.9 & $0.3-3.5$ \\
\hline OR = odds ratio; Cl = confidence interval & & & & & \\
\hline
\end{tabular}

commonly reported treatments were orthodontic treatments (13.1\%) and the placement of crowns (5.8\%).

Of all respondents, 74 (8.6\%) indicated to consider cosmetic dental treatment in the coming year. Whitening of teeth (3.2\% of the total sample), followed by orthodontic treatment (1.5\%) appeared to be the most popular procedures (see Table 1). No differences were found with regard to gender or age between those who did and who did not indicate to consider cosmetic dental treatment in the coming year.

\section{Cosmetic treatments and their relationship with characteristics of BDD}

Seventy subjects (8.0\%) were preoccupied with a defect in their appearance, while $1.5 \%(n=13)$ fulfilled all criteria for a positive diagnostic screening of BDD.

To determine the strength of the associations between any of the BDD characteristics and type of cosmetic treatment a series of logistic regression analyses were performed. It was found that individuals who reported a preoccupation with a defect in appearance did not differ from those who did not report this symptom in terms of having had any cosmetic dental treatment in the past $(\mathrm{OR}=1.2$; 95\% CI, 0.7-2.0). For none of the cosmetic procedures in the past preoccupation with a defect in appearance was a significant predictor (see Table 1).

Table 2 shows the prevalence of symptoms of BDD among patients who reported the intention to undergo cosmetic treatments in the coming year, as well as information concerning the strength of the associations (OR) between this intention and BDD characteristics. Those who showed a preoccupation with a defect in appearance were 6.6 times more likely to consider any form of cosmetic dental treatment in the coming year than those who did not display this BDD symptom (95\% CI, 3.7-11.8). Those who indicated to consider undergoing cosmetic dental treatment in the coming year, compared to those who did not report such intention, did not differ in their report on the remaining BDD features.

Therefore, a series of univariate logistic regression analyses were performed with the presence of a preoccupation with a defect in physical appearance as independent variable, and type of treatment in the past as dependent variable. These analyses revealed the presence of a preoccupation of a defect in appearance as a significant predictor of planned tooth whitening $(\mathrm{OR}=9.2,95 \% \mathrm{CI}, 4.1-$ 20.8), and of planned orthodontic treatment $(\mathrm{OR}=6.1,95 \% \mathrm{CI}, 1.8-20.6)$, but not of planned crowns (OR $=1.2,95 \% \mathrm{CI}$, 0.2-9.1). Odds ratios for the other types of planned dental procedures could not be computed due to the low prevalence rates of these treatments (see Table 1).

Furthermore, it was found that those who reported a preoccupation with their appearance were significantly more likely to be dissatisfied about their most recent cosmetic dental treatment than those who did not report this symptom $[28.6 \%$ versus $7.8 \%$; OR $=4.6 ; 95 \% \mathrm{CI}$, 1.3-16.5].

Multivariable logistic regression analyses were used to estimate the odds for (1) having undergone one or more cosmetic treatments in the past and (2) the intention of undergoing cosmetic treatment in the coming year associated with the sociodemographic variables, 
report of previous cosmetic treatments, and characteristics of BDD. As shown in Table 3, of these five variables only age was predictive of past cosmetic dental treatments while controlling for all other independent variables in the equation [goodness-of-fit $\chi^{2}=3.6$, $\mathrm{df}=6, \mathrm{p}=0.73$ ].

Two other variables were uniquely predictive of having the intention to undergo cosmetic dental treatment in the coming year while controlling for all other independent variables in the equation. These were: having undergone cosmetic treatment in the past and preoccupation with a defect in physical appearance [goodness-of-fit $\chi^{2}=10.7$, $\mathrm{df}=8, \mathrm{p}=0.22]$.

\section{DISCUSSION}

Interest in improving facial aesthetics or even being dissatisfied with one's own appearance does not necessarily have to be a problem. It does become problematic when the individual develops a preoccupation which leads to significant disruption in daily functioning and negative effects on his well-being and quality of life. Therefore, it is important to distinguish between functional or 'normal' forms of dissatisfaction about one's physical appearance, and dysfunctional or pathological forms of dissatisfaction such as BDD. To the best of our knowledge the present study was the first empirical investigation of presence of dysmorphic concern in relation to interest in, or motivation to involve in cosmetic dentistry.

The results of the present study did not find support for a relationship between a positive diagnostic screen of BDD and aesthetically motivated dental treatments. Although a sizeable sample was used, only one of the three diagnostic criteria for BDD discriminated between respondents who considered future cosmetic treatments and those who did not. Perhaps the sample has still been too small for a less common psychiatric disorder such as BDD. Also the fact that subjects who did not meet the preoccupation criterion were not requested to answer the remaining items (ie the other BDD criteria) might have limited the response. This, in turn, may have decreased the power to detect

Table 3 Results of multivariable logistic regression analyses with the factors being uniquely predictive for interest in cosmetic dental treatment (past treatments and future plans)

\begin{tabular}{l|l|l|l|l|} 
& \multicolumn{2}{|l|}{$\begin{array}{l}\text { Past cosmetic dental } \\
\text { treatments }\end{array}$} & \multicolumn{2}{l}{$\begin{array}{l}\text { Plans for cosmetic } \\
\text { dental treatment in } \\
\text { the coming year }\end{array}$} \\
\cline { 2 - 5 } Variable & OR & $95 \% \mathrm{Cl}$ & OR & $95 \mathrm{Cl}$ \\
\hline Being younger than 30 years & 1.8 & $1.3-2.5$ & & \\
\hline Having had cosmetic treatments in the past & & & 4.7 & $2.8-7.9$ \\
\hline Preoccupation with one's physical appearance & & & 6.3 & $3.3-12.2$ \\
\hline OR =odds ratio; $\mathrm{Cl}=$ confidence interval & & & & \\
\hline
\end{tabular}

meaningful differences related to the other BDD criteria between both groups.

Only one of the BDD features, a preoccupation with a defect of appearance, emerged as a significant predictor of undergoing cosmetic dental treatments, but only in relation to planned treatments and not to treatments in the past. More specifically, compared to those who did not report a preoccupation with a defect in appearance, those who indicated to fulfil this BDD feature were almost nine times more likely to consider tooth whitening, and six times more likely to consider orthodontic treatment in the near future. It may not be a coincidence that tooth whitening and orthodontic treatment were not only the most popular treatments in terms of future intentions, but also the types of treatments most strongly associated with significant concerns about physical appearance. The increased emphasis on physical perfection and TV programmes that portray the possibilities of surgical and dental procedures may not only cause general body image dissatisfaction but also create new markets with more people being prepared and willing to improve their physical appearance. This development may explain the results of the multivariate regression analysis, showing that a preoccupation with an imagined defect in physical appearance was only predictive of future cosmetic treatments and not of past treatments.

Preoccupation with one's physical appearance not only appeared to be the most important predictor of patients' intentions to undergo cosmetic dental treatment in the near future, it would also seem that it significantly increases the likelihood of dissatisfaction about the outcome of treatment. These findings are in accordance with results of studies on various areas of cosmetic surgery, showing that a significant proportion of patients with BDD, who undergo plastic surgical procedures, do not benefit from such procedures and report a high degree of dissatisfaction. ${ }^{10-12}$ Therefore, the present finding supports the notion that treatment does not necessarily improve the appearance concerns of patients presenting for cosmetic dental procedures.

The results of the present study should be interpreted cautiously. Firstly, one drawback of the study may be its sample selection, where individuals within the public domain were asked for participation in the survey. Such a sample could be selected with regard to one or more parameters, thus implicating a weak representativity and generalisability of the results. However, it should be noted that the main purpose of the present study was to test the hypothesis whether increased report of BDD symptoms would be associated with a greater interest in aesthetically motivated dental treatments, rather than determining the prevalence rate of BDD in the Dutch population. Yet, using distinct DSM-IV-TR-based criteria for the assessment of BDD the prevalence rate for current BDD found in the present sample was $1.5 \%$. This is in agreement with the prevalence rate of $1.7 \%$ found in the only published study on BDD using a representative sample of the general population and essentially the same set of BDD criteria. ${ }^{8}$ Also, the rate of $8 \%$ meeting the hallmark BDD criterion of being preoccupied with a perceived flaw in appearance obtained in this study comes close to the figure reported by Rief and his colleagues (10\%). ${ }^{8}$ Another possible drawback pertains to the question whether all reported treatments 
were indeed cosmetic. Although the participants were explicitly asked about treatments with a cosmetic purpose it cannot be ruled out that, for instance with regard to the decision of initiating orthodontic treatment in the past, also other factors (eg parents' decisions and requests) have played a role. This also holds true for other treatments such as those involving crowns, and facings. This was the reason that anterior composite restorations were not included as treatment type. It was assumed that for these types of treatments it would have been even more difficult to evaluate as to why it was decided upon to have these restorations made, and whether treatment was based upon dental trauma or caries rather than upon a cosmetic request. These limitations particularly pertain to the retrospective side of the data gathering, and may be less relevant for the information collected from patients about their future treatment plans. The average age of the participants of almost 34 years makes it likely that most of them were well able to convey the real answers as to the underlying reasons for their treatment request. In addition, the finding that the presence of a preoccupation with one's physical appearance was a significant predictor of the intention to initiate orthodontic treatment is consistent with research showing that dissatisfaction regarding one's appearance is one of the most important motivating factors for undergoing orthodontic treatment. ${ }^{21}$ Thirdly, assessment of psychiatric symptoms such as BDD characteristics relies heavily on the likelihood of participants' agreement to participate and to report on these matters. For example, it is likely that the report on BDD characteristics may have been impaired by shame and embarrassment to disclose the severity of individuals' preoccupation, which in turn may have led to underreporting, and therefore to an underestimation of the prevalence of symptoms of BDD. There- fore, the actual proportion of individuals with BDD characteristics among visitors of cosmetic clinics may be higher than is suggested by the results of the present investigation. Finally, attitude rather than behaviour was measured. In other words, people who indicated that they would consider cosmetic dental procedures in the near future may eventually decide not to undergo such treatments, because of costs or other reasons.

At present, it is unclear to what extent cosmetic enhancing of dental patients with characteristics of BDD actually has negative consequences for those undergoing it. Yet, it may be warranted to be vigilant to the potential presence of disproportionate appearance concerns, particularly in the case of comprehensive, cosmetic treatment plans, Firstly, since BDD sufferers frequently seek cosmetic procedures as a means of alleviating their distress ${ }^{9,12}$ and secondly, because there is evidence to suggest that patients with characteristics of BDD are more likely to remain chronically dissatisfied about their treatment. ${ }^{11,12}$ Therefore, the next research endeavour in this area should be a study among those who attend dental clinics for an aesthetically motivated dental treatment. It would be enlightening to examine whether the proportion of patients with features of BDD is higher than in the general population, and whether these individuals display more dissatisfaction, for example concerning the shape, size or some other aspect of the appearance (eg asymmetry or disproportion of the teeth or the face) than those not displaying these features. Valuable information could be obtained by using a longitudinal sample. Such a design would provide information about continuity and change in both BDD symptomatology, satisfaction about cosmetic treatments, general well-being, and quality of life.

1. Sarwer D B, Grossbart T A, Didie E R. Beauty and society. In Kaminer M S, Dover J S, Arndt J K (eds).
Atlas of cosmetic surgery. pp 48-59. Philadelphia: Saunders, 2002.

2. American Society for Aesthetic Plastic Surgery. Cosmetic Surgery National Data Bank 2006 Statistics. New York: American Society for Aesthetic Plastic Surgery, 2007.

3. Crerand C E, Franklin M E, Sarwer D B. Body dismorphic disorder and cosmetic surgery. Plast Reconstr Surg 2006; 118: 167-180.

4. Sarwer D B, Wadden T A, Pertschuk M J, Whitaker $L A$. Body image dissatisfaction and body dysmorphic disorder in 100 cosmetic surgery patients. Plast Reconstr Surg 1998; 101: 1644-1649.

5. American Psychiatric Association. Diagnostic and statistical manual of mental disorders (DSMIV-TR). Washington, DC: American Psychiatric Association, 2000.

6. Corove M B, Gleaves D H. Body dysmorphic disorder: a review of conceptualizations, assessment, and treatment strategies. Clin Psychol Rev 2001; 21: 949-970

7. Oosthuizen P, Lambert T, Castle D J. Dysmorphic concern: prevalence and association with clinical variables. Aust N Z J Psychiatry 1998; 32: 171-179.

8. Rief W, Buhlmann U, Wilhelm S, Borkenhagen A, Brahler E. The prevalence of body dysmorphic disorder: a population-based survey. Psychol Med 2006; 36: 877-885.

9. Philips K A, McElroy S L, Keck P E Jr, Pope H G Jr, Hudson J L. Body dysmorphic disorder: 30 cases of imagined ugliness. Am J Psychiatry 1993; 150: 302-308.

10. Veale D, Boocock A, Gournay K et al. Body dysmorphic disorder: a survey of fifty cases. Br J Psychiatry 1996; 169: 196-201.

11. Mackley C L. Body dysmorphic disorder. Dermatol Surg 2005; 31: 553-558.

12. Veale D. Outcome of cosmetic surgery and 'DIY' surgery in patients with body dysmorphic disorder Psychol Bull 2000; 24: 218-221.

13. Uzun O, Basoglu C, Akar A et al. Body dysmorphic disorder in patients with acne. Compr Psychiatry 2003; 44: 415-419.

14. De Jongh A, Adair P. Mental disorders in dental practice: a case report of body dysmorphic disorder. Spec Care Dent 2004; 24: 61-64.

15. Herren C, Armentrout T, Higgins M. Body dysmorphic disorder: diagnosis and treatment. Gen Dent 2003; 51: 164-166.

16. Cunningham S J, Bryant C J, Manisali M, Hunt N P, Feinmann C. Dysmorphophobia: recent developments of interest to the maxillofacial surgeon. Br J Oral Maxillofac Surg 1996; 34: 368-374.

17. Rispoli A, Acocella A, Pavone I et al. Psychoemotional assessment changes in patients treated with orthognathic surgery: pre and postsurgery report. World J Orthod 2004; 55: 48-56.

18. Cunningham S J, Feinmann C. Psychological assessment of patients requesting orthognathic surgery and the relevance of body dysmorphic disorder. Br J Orthod 1998; 25: 293-298.

19. Van Rood $Y R$, Den Hollander-Gijsman M E, De Jongh A, De Beurs E. Development and validation of a screening instrument for body dysmorphic disorder, the SI-BDD. Submitted for publication.

20. Dutch Central Bureau of Statistics. Population figures. Heerlen: Centraal Bureau voor de Statistiek, 2005. http://statline.cbs.nl/StatWeb

21. Bos A, Hoogstraten J, Prahl-Andersen B. Expectations of treatment and satisfaction with dentofacial appearance in orthodontic patients. Am J Orthod Dentofacial Orthop 2003; 123: 127-132. 